\title{
TEACHING FRENCH LANGUAGE AND CULTURE IN MACEDONIA - A TRUE CHALLENGE
}

\author{
[ENSEIGNER LA LANGUE ET LA CULTURE FRANCAISES EN \\ REPUBLIQUE DE MACEDOINE : UN VERITABLE DEFI]
}

\author{
Snezana Petrova
}

\section{DOI: 10.18355/PG.2015.4.2.172-180}

\begin{abstract}
The knowledge and the practice of the French language and culture of yore, gave you a certain position in the Macedonian society. It gave you a certain pride, made you prominent, renowned and influential. But that stopped being the case in these recent years and especially today. Nowadays, learning and teaching this language are not relevant, and so, they are less sustained. To better present you with the problematic, we addressed our study with a little history about the teaching of the language to lead to an embodiment and a reflection of it is current situation in our primary schools, high schools and universities in Macedonia. Finally, we can see the actions that have been undertaken to improve the situation.
\end{abstract}

\section{Keywords}

French, language, culture, education, history, current events, awareness

\section{Résumé}

La connaissance et la pratique de la langue et culture françaises, d'antan, vous donnaient une position dans la société macédonienne, vous insufflaient un certain orgueil, vous faisaient valoir, mais ce n'est plus le cas ces dernières années et particulièrement aujourd'hui. Actuellement, l'apprentissage et l'enseignement de cette langue ne sont plus vraiment d'actualité, plus aussi soutenus et pour pouvoir mieux vous représenter la problématique nous avons abordé notre étude par un petit historique de l'enseignement de cette langue pour ensuite aboutir à une concrétisation et une réflexion de la situation actuelle de l'enseignement et de l'apprentissage de la langue française dans les écoles primaires, lycées, universités en Macédoine et finalement voir les actions qui ont été entreprises pour améliorer la situation.

\section{Mots clés}

le français, la langue, la culture, l'enseignement, historique, faits actuels, la sensibilisation

Hors des frontières françaises, il y a beaucoup de francophones et de francophiles qui jouent un rôle prépondérant dans l'expansion de la culture et langue françaises sur leur territoire. Ainsi cette langue qui est la $5^{\mathrm{e}}$ langue la plus parlée au monde, la troisième langue des affaires à l'échelon international, et celle que parleront bientôt plus de 350 millions de jeunes 
d'Afrique (il est prévu que le nombre de francophones approchera les 700 millions en 2050 et $85 \%$ de ces francophones seront en Afrique) est plus ou moins présente sur plusieurs pôles en Macédoine; économique, politique, culturel, mais celui sur lequel je voudrais m'attarder, c'est celui de l'éducation plus exactement de l'enseignement de et en français.

\section{La présence du français dans le système éducatif macédonien - historique et faits actuels}

La France au travers de sa langue et culture comme sous le sigle de la Francophonie était très bien représentée sur le sol macédonien. Chronologiquement, au 19e siècle, le français était vu comme une langue diplomatique et de prestige qui était parfaitement connue par les familles privilégiées lesquelles n'hésitaient pas à envoyer leurs enfants étudier dans de grandes écoles ou universités en France. Plus tard, jusqu'à la Seconde guerre mondiale, le français était enseigné dans des collèges et lycées français tenus par des religieuses.

Cette place de prépondérance était encore visible en 1994 après la proclamation de l'indépendance de la Macédoine en 1991 et selon les statistiques, le français était représenté à $35 \%$ au niveau des écoles primaires, juste derrière l'anglais, mais devant le russe et l'allemand lequel ne comptait que $2 \%$. Cette même seconde place était aussi assurée dans l'enseignement secondaire.

Fait important est qu'en 1997, des sections bilingues avec le français ont été mises en place dans quelques lycées de Macédoine (dans des lycées de la ville de Kumanovo, de Tetovo, de Skopje - avec 2 lycées bilingues, un lycée professionnel, comme une école primaire, de Bitola et de Negotino). Il s'agit donc de sections où certaines matières (de une à quatre : cela va progressivement) sont enseignées en français au cours des quatre ans d'étude que constitue l'enseignement secondaire selon le système éducatif macédonien. A la fin de ces études, les élèves ont la possibilité d'acquérir un certificat de maîtrise de la langue française délivré par l'ambassade de France après avoir passé un examen final en français sur les quatre matières qu'ils ont étudiées au cours de leur quatre années d'enseignement secondaire dont le français. Il faut savoir que cet enseignement bilingue facilite l'accès à des universités étrangères (cependant certaines universités françaises demandent en plus un certificat ou diplôme d'étude en langue française ; niveau B2 ou même $\mathrm{C} 1$, pour prouver le niveau de compétence en langue française du candidat) et est un excellent moyen d'ouverture sur l'Europe et le monde.

Plus tard en 1999, s'est ouvert le premier jardin d'enfants francophone privé à Skopje lequel assurait un enseignement de la langue française en tant que langue maternelle et non pas en tant que langue étrangère. Celui-ci comportait toutes les classes de maternelle plus exactement la petite, la moyenne et la grande section. Ce jardin d'enfants était soutenu par l'Ambassade de France et fournissait un enseignement à la française de grande qualité. Puis quelques années plus tard, un autre jardin d'enfants

Slavonic Pedagogical Studies Journal Vol. 4 Issue 2, September 2015 
français privé s'est ouvert dans la même ville "Dr Judith et Dr Joseph" qui assure aujourd'hui encore un bon programme d'éducation en langue française mais qui ne possède pas le label de maternelle ou école française où les enseignements sont reconnus conformes aux programmes français.

A la rentrée 2014, une métamorphose de l'ancien jardin d'enfants francophone de Skopje s'est produite. De simple jardin d'enfants, il est devenu une véritable école française qui propose non seulement toutes les sections de la maternelle mais offre en plus, l'ensemble du cycle de l'école primaire française jusqu'au CM2. L'Ecole Française Internationale de Skopje (EFIS) est dirigée par un directeur français, titulaire de l'Education nationale, M. Sébastien Chevallet. Attestés par le Ministère français de l'Education nationale, les enseignements y sont reconnus conformes aux programmes français ainsi qu'aux pratiques pédagogiques modernes. L'EFIS a entre autre signé une convention avec la Mission laïque française, organisme officiel d'encadrement et de contrôle des écoles françaises à l'étranger, plus exactement avec M. Jean-François Le Roch qui en est devenu le gérant et s'est engagé à faire de cette école un établissement scolaire complet qui en fin de cycle délivrera un baccalauréat français ou international. Assurément le nombre d'enfants inscrits est en augmentation mais il est clair que c'est un enseignement privé et qui donc a un certain coût pour les parents.

Dans l'enseignement supérieur, l'apprentissage de la langue, culture et littérature françaises est aussi présent :

- à l'université "Saints Cyrille et Méthode" à Skopje, plus exactement à la faculté de philologie «Blaze Koneski », au département de langues et littératures romanes.

Ce département a été ouvert en 1946, année de conception de la faculté de philosophie, et portait auparavant le nom de «Département de philologie romane » au sein duquel se trouvait le groupe d'étude de langue et littérature françaises. Puis en 1974/75, les groupes d'études philologiques de la faculté de philosophie se sont regroupées, se sont détachées, pour constituer la faculté de philologie «Blaze Koneski ». $\mathrm{Au}$ sein de cette faculté, actuellement il y a une vingtaine de départements dont le département de langues et littératures romanes et le département de traduction et d'interprétation où est enseigné le français. Au premier département, on ne compte plus qu'une petite vingtaine d'étudiants en français (en 2013, 2014, 2015) en première année, tandis qu'au département de traduction et d'interprétation on comptabilise en 2014/2015 près de 10 élèves qui choisissent le français en tant que première langue étrangère (langue active) et entre 30 et 35 élèves qui choisissent le français en tant que seconde langue étrangère (langue passive). Pour augmenter le nombre d'étudiants en première année au département de langues et littératures romanes, il a été décidé depuis peu qu'il serait aussi possible d'accepter de vrais débutants en langue française alors que depuis des décennies, les nouveaux étudiants devaient avoir obligatoirement étudié le français dans le secondaire.

- à l'université d'Etat de Tetovo (formée en 1994), faculté de philologie ; 
département de langues et littératures romanes qui comptent en 2015 près de 18 étudiants en première année.

- dans d'autres universités et facultés du pays mais en tant que matière optionnelle ou facultative.

Le Ministère de l'éducation et des sciences de la République de Macédoine, suite aux recommandations du Conseil de l'Europe, depuis quelques années, poursuit une politique en faveur de l'apprentissage de deux langues étrangères dans le primaire et c'est ainsi qu'en 2007, une nouvelle réforme dans l'éducation instaure l'anglais en tant que première langue étrangère obligatoire. Le résultat de cette réforme est qu'à l'avenir il ne sera plus possible de choisir le français en tant que première langue étrangère mais uniquement en seconde - exclusion faite pour les lycées bilingues. Ceci fait que depuis 2007, dans les écoles primaires, la seconde place est disputée entre le français, l'allemand, le russe et dernièrement l'italien. Plus concrètement, la seconde place qui pendant des années était assurée pour le français, lui a été retirée en 2014 au profit de l'allemand. Le choix de la seconde langue étrangère qui se fait à la fin de la $5^{\mathrm{e}}$ classe (CM2 selon le système français) est à priori fait par les élèves/parents (demande ou fiche d'enquête qui doit être complétée par les parents pour informer l'école de leur choix de langues), mais sur le terrain les choses sont différentes car ce choix est plutôt fait par le Maire de la ville ou de la commune ou bien encore par les directeurs d'école qui décident de quelle langue sera étudiée dans leur établissement.

Actuellement l'apprentissage du français dans les écoles primaires perd nettement du terrain dans les plus grandes agglomérations, finit par être enseigné que dans les écoles primaires de campagne et par effet de boule de neige, il devient de moins en moins enseigné dans les lycées comme à l'université. Mais chose encore plus alarmante est que le français qui était enseigné en tant que seconde langue étrangère dans les lycées professionnels, suite aux dernières réformes, va totalement disparaître à partir de la prochaine rentrée scolaire. Il n'y sera enseigné plus qu'une seule langue étrangère ; l'anglais, qui rappelez-vous est obligatoire.

Enseigner la langue française en Macédoine devient chose difficile et contraignante. Premièrement à cause de l'aide insuffisante donnée par l'Etat français - ce qui provoque une réaction plus ou moins négative des directeurs d'établissement comme des enseignants qui s'estiment lésés, alors qu'ils sont en train de faire valoir et promouvoir une langue et une culture qui n'est pas la leur. Il est clair que l'Etat français dans ces difficiles moments de restriction budgétaire doit faire des économies, mais de là à ne consacrer que $1,2 \%$ de son budget, me semble un peu trop tiré par les cheveux. Les autres grands réseaux de promotion de la langue et de la culture françaises que sont les deux Alliances françaises de Macédoine (de la ville de Bitola et de Tetovo) et l'Institut français de Skopje sont obligés de subvenir à leurs besoins par leurs propres moyens car le budget annuel qui leur est octroyé est considérablement réduit et donc insuffisant. Dans le projet de loi de finance 2005 publié sur le site Internet du Sénat, on pouvait

Slavonic Pedagogical Studies Journal Vol. 4 Issue 2, September 2015 
lire:

"Ce qui est troublant, [c'est] qu'il n'est jamais question de fermer une ambassade endormie mais que des centres culturels disparaissent alors que peu sont ouverts [...] En asphyxiant ces Centres et Instituts progressivement avant de les déclarer moribonds et inutiles, on sacrifie un des leviers de la diplomatie culturelle de la France dont on continue de faire grand cas dans les discours, mais surtout dans les discours." ${ }^{1}$

En 2015, non seulement l'Institut et les alliances françaises perdent de leur personnel mais ils pourraient aussi risquer la fermeture par manque de finances. Ce serait une chose désastreuse pour l'enseignement et l'apprentissage de la langue et culture françaises dans notre pays. Une présence, une visibilité et des actions de la France ou de pays francophones sont plus que primordiales.

Voici quelle est la situation et de quelle manière elle est ressentie. Que pouvons nous faire ? Y a-t-il une solution ? Que faisons nous pour sortir de cette impasse?

Malgré cet aspect plutôt administratif et plus ou moins décourageant des choses, enseigner le français est encore un plaisir que nous voulons partager avec nos élèves ou futurs enseignants. Ainsi l'Association des professeurs de français de la république de Macédoine en collaboration avec la Commission nationale pour la Francophonie, l'Institut français de Skopje sans oublier les Alliances, depuis 2014, a lancé le projet de sensibilisation à la langue française dans les petites classes. J'entends par petite classe, les $5^{\mathrm{e}}$ classes selon le système d'éducation macédonien. Cette année d'enseignement est notre cible, car l'année suivante (la $6^{\mathrm{e}}$ classe) est celle où les élèves/parents font le choix de la seconde langue étrangère. Ainsi des activités ludiques, des jeux, des chansons etc... sont offerts aux élèves intéressés et tenus de façon bénévole par des professeurs ou des futurs professeurs de français au sein même des écoles où il n'y a pas d'enseignement du français ou dans les écoles où l'enseignement du français est assuré mais s'est essoufflé avec le temps. Quoiqu'il en soit, nous faisons parler de nous et de la langue et culture françaises. Nous luttons pour la remettre au goût du jour, pour la rendre encore plus intéressante, attirante, ce qui n'est pas forcément au goût des directeurs d'établissements et des maires des villes. Nous n'avons pas encore récolté les fruits de notre labeur; nous attendons avec impatience les premiers signes à la prochaine rentrée scolaire. Notre intention est de continuer avec ce projet, de l'étendre dans le plus de villes possibles, dans le plus grand nombre d'établissements, et nous ne perdons pas espoir sachant que ces changements ne peuvent s'effectuer du jour au lendemain.

\section{L'enseignement du français : les enseignants, les méthodes et manuels}

La langue française est donc enseignée en tant que deuxième langue étrangère dans l'enseignement primaire de la 6e classe à la neuvième qui est la dernière classe du primaire selon le système éducatif macédonien.

${ }^{1}$ Puren, C. (1988): Op. cit., p.44 
Dans le secondaire général ou bilingue, elle est enseignée de la première à la quatrième et dernière année du secondaire et porte le statut de matière obligatoire et dans les lycées professionnels à partir de la rentrée 2015, elle ne sera plus du tout enseignée.

Les enseignants qui s'occupe de cet enseignement de la langue française doivent avoir un diplôme adéquat de professeur de français obtenus au terme de 4 ans d'études et après la soutenance d'une thèse - diplôme qui est délivré par l'Université "Saints Cyrille et Méthode" ou bien par l'Université d'Etat de la ville de Tetovo, posséder un niveau C1, C2 en français mais aussi de solides compétences pédagogiques, psychologiques, professionnelles et méthodologiques.

Les enseignants de DNL des classes bilingues, quant à eux, doivent avoir au moins le niveau B2 dans leur connaissance de la langue française pour pouvoir enseigné leur matière en français.

En ce qui concerne les programmes d'enseignement, les enseignants des établissements primaires et secondaires de la République de Macédoine suivent les directives des programmes d'enseignement officiels délivrés par le Ministère de l'éducation et des sciences dans lesquels il y a les objectifs, les contenus d'enseignement de la matière, les activités et les standards d'évaluation comme d'autres éléments prévus pour compléter le travail de l'enseignant.

Les programmes d'enseignement pour le primaire comme pour le secondaire suivent les directives du Cadre européen commun de référence pour les langues.

A la fin de la première année d'apprentissage de la langue française dans le secondaire, il est possible d'atteindre le niveau $\mathrm{A} 2$, à la fin de la deuxième année d'étude ; A2+, fin de troisième année; B1 partiel et à la fin de la 4e et dernière année niveau B1 complet.

Les manuels de français qui sont utilisées pour l'enseignement de la langue sont choisis et surtout agréés par une commission engagée par le ministère de l'éducation et des sciences pour servir à cet effet. Ainsi dans les établissements primaires sont utilisés actuellement les manuels de français suivants:

En 6e classe: $M a g$

En 7e classe : Vite 2

En 8e classe: Vite 3

En 9e classe: Pixel 4

Dans les établissements secondaires généraux sont utilisés :

Campus 1 et 2 et Café Crème 1 et 2.

Dans les classes bilingues sont utilisés Campus 1 et 2 ainsi que le manuel Reflet.

Dans l'enseignement secondaire professionnel sont utilisés Nouveau Taxi 1, 2, 3 mais aussi Belleville, Café Crème 1 et 2. ${ }^{1}$

${ }^{1}$ Cependant, selon les nouveaux programmes d'enseignement, pour la prochaine rentrée scolaire, le ministère de l'éducation et des sciences ainsi que le bureau de 
Dans la pratique, durant ces dernières années, en ce qui concerne les méthodologies d'enseignement, on peut dire qu'elles ne sont pas uniques et universelles. Différents enseignants dans différentes écoles utilisent différentes méthodologies d'enseignement tendant à une diversification des matériels et des approches et rechignent à tout ce qui est imposé. Majoritairement, ils n'optent plus pour un suivi aveugle des méthodologies d'enseignement actuelles ou bien des manuels mais ils les adaptent, les multiplient, les ajuste gardant comme point de mire les directives du programme officiel d'enseignement qui est élaboré et délivré par le bureau de développement de l'éducation. Ceci est plus ou moins critiquable, mais de nombreux professeurs préfèrent adopter ou sont obligés d'adopter la photocopieuse à la place du manuel et de cette façon se construisent une méthodologie qui leur est propre, des cours de langue qui répondent à la demande des apprenants et surtout au niveau et au statut du groupe d'apprenants. Les bienfaits de cette méthodologie individuelle ont été prouvés car elle amène de façon plus rapide et plus efficace à des prises de parole, à la communication en langue française plus ouverte, moins timide et à des élèves moins récalcitrants envers l'apprentissage de cette langue.

\section{L'insécurité des enseignants}

Grâce à de nombreux contacts avec les enseignants, je me suis rendue compte qu'une certaine insécurité linguistique ou langagière existe ou apparaît avec le temps chez certains d'entre eux. J'entends par insécurité linguistique ce manque de facilité dans la communication qui fait que l'enseignant hésite à communiquer ou à s'adresser en français avec un locuteur natif de peur de paraître ridicule ou pas suffisamment expérimenté. Beaucoup d'enseignants tiennent leurs cours avec un lexique assez restreint et font tout leur possible pour ne pas laisser paraître un semblant de manque de connaissance de la langue ou de professionnalisme. En fait, l'insécurité linguistique se place particulièrement dans les besoins quotidiens, dans des situations de communication réelles, avec des collègues, des personnes de plus hautes hiérarchies et donc d'une meilleure connaissance linguistique et lexicale ou bien avec des natifs, des touristes ou amis francophones. En gros, il s'agit ici d'une certaine peur, d'une honte et l'impossibilité de dire ce que l'on veut dire par des mots simples en français. Ce problème est ou peut devenir réel, mais je ne dis pas qu'il est récurant à tous les professeurs. Le manque de pratique de la langue provient dans certains cas de l'insuffisant niveau atteint à la fin des études, de l'absence de pratique de la langue par la suite, de la répétition des mêmes cours ou unités d'enseignement, du manque de contact entre collègues en langue française, de l'impossibilité ou la mauvaise volonté de certains enseignants de suivre des cours de remédiation ou simplement des cours à distance (je peux aussi

développement de l'éducation prévoient que dans les classes primaires et secondaires il sera utilisé un seul et même manuel par année d'étude et pour tous les types d'enseignement (classique, bilingue, professionnel). 
comprendre un grand nombre d'entre eux car nous sommes tous en tant qu'enseignants de plus en plus noyés dans la paperasse administrative, ce qui ne nous laisse que peu de temps pour nous remettre à niveau ou simplement jouir du français par différents moyens). La seule solution à cette insécurité linguistique serait entre autre une remédiation linguistique régulière, de plusieurs semaines ; un bain linguistique régulier mais aussi particulier ou individuel en France ou dans un pays francophone qui serait organisé et principalement assuré financièrement par l'établissement scolaire auquel appartient l'enseignant ou par d'autres institutions ou organismes.

\section{Conclusion}

Selon le registre des Statistiques de la République de Macédoine et les données que l'on peut y trouver sur l'apprentissage des langues étrangères et surtout du français nous pouvons voir qu'en 2014, nous avons perdu la seconde place dans l'enseignement des langues étrangères au profit de l'allemand. L'enseignement de la langue française est en net recul. De moins en moins d'élèves sont intéressés par cet apprentissage et préfère l'allemand au français pour des causes économiques; ils pensent qu'ils peuvent trouver plus facilement un emploi avec cette langue car les entreprises qui investissent dans notre pays sont majoritairement des entreprises allemandes et non françaises. D'autres élèves préfèrent même l'italien au français et lorsqu'on leur pose la question sur leur choix, ils nous disent que l'italien est plus facile à apprendre et qu'il est plus mélodieux. Le résultat de ces choix fait qu'un nombre assez important d'enseignants de français voient l'effectif de leur classe diminué et même certaines classes disparaissent. Certains professeurs ont déjà perdu ou vont perdre prochainement leur emploi, d'autres qui partent en retraite voient leur poste non remplacé et le français se voit retiré du programme.

Notre réaction, entre autre, a été d'augmenter nos activités extra et intra scolaires, d'actualiser et dynamiser nos cours, d'attirer l'attention le plus possible en offrant une sensibilisation de la langue française dans les écoles primaires, d'inventer et de prolonger avec l'organisation de concours qui seraient médiatisés, comme le concours de la dictée et le concours d'orthographe, les Olympiades, etc., d'instaurer ou de faire participer nos élèves à des camps d'été où le français serait la langue de communication, faciliter et organiser des séjours linguistiques, des formations dans des pays francophones non seulement pour les élèves apprenants mais aussi pour les enseignants.

L'amour pour la langue française subsiste encore en Macédoine mais il faut le régénérer et c'est ici que nous trouvons notre place, nous, professeurs ou

1 Cette remédiation ne devrait pas entièrement être à la charge de l'enseignant, car nous savons tous que le salaire d'enseignant ne peut aucunement couvrir de façon régulière ce genre de formation.

Slavonic Pedagogical Studies Journal Vol. 4 Issue 2, September 2015 
enseignants, francophones et francophiles, en tant que grand amoureux de cette langue. Et moi en tant que fondatrice du premier jardin d'enfants francophone à Skopje et en tant que professeur universitaire à l'université Saints Cyrille et Méthode, j'estime que nous pouvons encore faire bouger les choses.

\section{Bibliography}

LENNEBERG, E.H. 1967. Biological foundations of language. New York: Wilep. Available online: www.lel.ed.ac.uk/ jim/crit.per.pdf - http://apf.pcf.be/ROOT/apf/enseignement_francais.html

BARTHELEMY, F. - GROUX, D. - PORCHER, L.: Cent mots pour le français langue etrangere. Paris: L'Harmattan. ISBN 9782296545021.

CASTEllotTI, V. - De CARLO, M. - GALISSON, R. 1995. La formation des enseignants de langue. Paris: Cle international. ISBN 2090332498.

DEFAYS, J. M. 2003. Le francais langue etrangere et seconde. Enseignement et apprentissage. Belgium: Mardaga. ISBN 2870098464.

GALISSON, R. 1980. D'hier a aujourd'hui, la didactique generale des langues etrangeres. Paris : Cle international. ISBN 2190332567.

GALISSON, R. - PUREN, C. 1999. La formation en questions. Didactique des langues etrangeres. Paris: Cle international. ISBN 2090333308.

GALISSON, R. 1995. "A enseignant nouveau, outils nouveaux", Le francais dans le monde (recherches et applications), Numero special "Methodes et methodologies", janvier, pp.70-78.

Sources available online :

http://www.unige.ch/traduction-

interpretation/recherches/groupes/elf/medias/arbeitsmarkt_1007.pdf

-http://www.scribd.com/doc/80390629/Билингвална-настава-на-

француски-јазик-во-Р-Македонија-и-Гимназијата-,Орце-Николов“-

Скопје

http://www.google.com/search?client=safari\&rls=en\&q=државни+службе

ници+од+министерствата+на+P.M+и+странските+јазици\&ie=UTF-

$8 \&$ oe $=\mathrm{UTF}-8$

Prof. Snezana Petrova

Sts. Cyril and Methodius University

Faculty of Arts Blaze Koneski

Department of Romance languages and literatures

50 Divizija 6, 1000 Skopje

Macedonia

snezanapetrov@me.com 